\title{
Fibromialgia: Un diagnóstico que debe ser oportuno
}

\author{
Fibromyalgia: A diagnosis that must be opportune
}

\author{
Alfredo Berrocal-Kasay ${ }^{1, a ; 2, b}$
}

\section{RESUMEN}

La Fibromialgia (FM) es una entidad caracterizada por dolor musculo esquelético difuso crónico que afecta mayormente mujeres entre los 30-50 años de edad. Además del dolor difuso los pacientes con FM presentan con frecuencia trastornos del sueño, depresión y fatiga. El manejo es no farmacológico (psicoterapia principalmente) y farmacológico, los antidepresivos juegan un rol importante y entre ellos dos inhibidores duales de la recaptación de serotonina y noradrenalina como la duloxetina y minalcipran han sido aprobados por la FDA de EEUU para el control del dolor crónico. Asimismo, drogas usadas para dolor neuropático y convulsiones también han mostrado eficacia en el tratamiento del dolor difuso crónico, una de ellas la pregabalina también ha sido aprobada por la FDA de EEUU para el tratamiento de FM. Cada vez, se reconoce con mayor frecuencia esta enfermedad, sin embargo aún falta difundir su cuadro clínico y principales comorbilidades asociadas entre los médicos del primer nivel de atención, para que su diagnóstico sea oportuno y el tratamiento lo más eficaz posible para mejorar la calidad de vida de estos pacientes.

PALABRAS CLAVE: Fibromialgia, depresión, dolor crónico (Fuente: DeCS BIREME)

\section{SUMMARY}

Fibromyalgia (FM) is a condition characterized by chronic diffuse musculoskeletal pain that affects mostly women between 30-50 years of age. In addition patients with fibromyalgia often present sleep disorders, depression and fatigue. The management is pharmacological and not pharmacological (mainly psychotherapy); antidepressants play an important role and including two dual inhibitor of serotonin and norepinephrine reuptake as duloxetine and minalcipran that have been approved by the FDA in USA, for the control of chronic pain. Also, drugs used for neuropathic pain and seizures have shown efficacy in the treatment of chronic widespread pain, pregabalin has been approved by the FDA for the treatment of FM. Each time, this disease is recognized more frequently; however, yet to spread its clinical picture and main comorbidities among physicians from primary care, to the diagnosis and appropriate treatment is as effective as possible to improve the quality of life of these patients.

KEYWORDS: Fibromyalgia, depression, chronic pain (Source: MeSH NLM)

\footnotetext{
Servicio de Reumatología, Hospital Nacional Cayetano Heredia (Ministerio de Salud). Lima, Perú. Facultad de Medicina Alberto Hurtado, Universidad Peruana Cayetano Heredia. Lima, Perú. 


\section{INTRODUCCIÓN}

La Fibromialgia (FM) es una enfermedad caracterizada por dolor musculo esquelético crónico; anteriormente se le conocía como fibrositis, sin embargo, la ausencia de inflamación en los estudios histológicos de músculos afectados alejó el uso de este término. Posteriormente, el Colegio Americano de Reumatología (ACR) acuñó el nombre de Fibromialgia (FM) y en 1990 publicó los criterios para su clasificación (1) (Tabla 1). En el 2010, el ACR publicó nuevos criterios preliminares para el diagnóstico de FM ; estos nuevos criterios también deben estar presentes por lo menos durante 3 meses pero no consideran los 18 puntos dolorosos clásicos, incorporando en cambio un índice de dolor difuso que cuantifica el dolor entre 0 (ausente) y 1 (presente) en 19 áreas durante la última semana así como una escala de severidad de síntomas como fatiga, sueño no reparador y síntomas cognitivos reportados también en la última semana (2). La FM afecta fundamentalmente mujeres entre 30 a 50 años de edad y en EEUU se estima que la prevalencia en población adulta es del 2\% (3); en el Perú, no existen estudios epidemiológicos, sin embargo, un estudio realizado en población urbano marginal de Lima reportó una prevalencia del 10\% (4).

\section{FISIOPATOLOGIA}

Algunos autores proponen que diversos factores genéticos y ambientales pueden estar relacionados en su origen. Algunos estudios sugieren asociación con alteraciones de diversos genes como el receptor de serotonina $2 \mathrm{a}$, la región regulatoria del transportador de serotonina o el receptor de dopamina D4 $(5,6)$.

Asimismo existen otros factores estresores como los problermas emocionales que ocurren en la vida adulta o que pudieron suceder en la infancia, como abuso físico, sexual, emocional o social que pueden estar relacionados a FM $(7,8)$.

Cada vez se evidencia más que en FM ocurre un procesamiento anormal del dolor existiendo mayores datos que sugieren una disminución de la inhibición de las fibras que conducen el dolor, así se explica que estos pacientes no sólo presenten dolor en los puntos gatillo característicos sino también en cualquier parte del cuerpo y que además tengan una respuesta dolorosa incrementada a estímulos que normalmente no causarían dolor (9-11).

\section{CUADRO CLINICO (12-15)}

La FM afecta mayormente mujeres entre los 30 a 50 años de edad .Lo que caracteriza a esta enfermedad es el dolor difuso que se asocia además a fatiga, insomnio y depresión.

El dolor puede ser nociceptivo o neuropático; aunque existen artralgias realmente no existe compromiso articular inflamatorio. Asimismo puede presentarse mialgias y parestesias sin que exista miopatía o neuropatía, algunas mujeres pueden presentar disuria o vejiga hiperactiva aunque no se documente infección u otra patología urinaria.

Tabla 1. Criterios del ACR 1990 para Fibromialgia.

1. Historia de dolor difuso de por lo menos 3 meses de duración.

2. Dolor en 11 de 18 puntos sensibles a la palpación digital. Esta debe ser con una fuerza aproximada de $4 \mathrm{~kg} \mathrm{y}$ para que el punto sensible sea considerado positivo el paciente debe señalar que la palpación fue dolorosa.

- Occipucio: dolor en la inserción del músculo suboccipital , bilateral

- Cervical bajo: aspecto anterior del espacio intertransversal entre C5 a C7, bilateral

- Trapecio: punto medio del borde superior, bilateral.

- Supraespinoso: encima de la espina escapular, cerca del borde medio, bilateral.

- Segunda costilla: a nivel de la segunda costocondral, bilateral

- Epicóndilo lateral: $2 \mathrm{~cm}$ distales a los epicóndilos, bilateral

- Glúteo: en cuadrante superior externo de las nalgas, bilateral

- Trocánter mayor: posterior a la prominencia trocantérica, bilateral

- Rodilla: en panículo graso medial proximal al espacio articular, bilateral 
Otros síntomas que también pueden verse en estos pacientes son pérdida de la memoria y la concentración, colon irritable, disminución de la capacidad para el ejercicio, cefalea; hasta en 10\% de casos se ha reportado fenómeno de Raynaud.

\section{LABORATORIO}

Tanto el hemograma como los reactantes de fase aguda son normales, se recomienda en estos pacientes determinar los niveles de hormonas tiroideas ya que el hipotiroidismo puede semejar este cuadro.

Es interesante señalar que alrededor del 10\% de los pacientes pueden desarrollar anticuerpos antinucleares (15), lo cual aunado al dolor difuso como artralgias puede conducir a un diagnóstico erróneo de lupus eritematoso sistémico.

También se ha reportado factor reumatoide positivo en menos del 10\% de pacientes aunque al igual que en el caso de los anticuerpos antinucleares los títulos son bajos. Hasta $50 \%$ de pacientes presentan anticuerpos antipolímero (APA), sin embargo no se recomienda utilizarlos para el diagnóstico de FM.

\section{TRATAMIENTO}

El manejo de FM es no farmacológico y farmacológico.

\section{Manejo no farmacológico (16-18)}

El dolor crónico en estos pacientes tiene un mecanismo multifactorial (emocional, genético, social, etc.), lo cual explica que no todos respondan adecuadamente al manejo con los diferentes medicamentos utilizados; por ello, se han diseñado diferentes estrategias como el manejo psicoeducativo para lograr que los pacientes comprendan su enfermedad y la interrelación entre el dolor y las alteraciones anímicas, del sueño y la fatiga. Otro enfoque es la terapia cognitiva-conductual que trata de erradicar los pensamientos y emociones negativas disminuyendo el distress emocional y la falta de autoestima a través de técnicas de refuerzo positivo y de disminución de pensamientos de mala adaptación logrando así la mejoría del ánimo y una disminución en la intensidad del dolor. Asimismo la utilización de técnicas de relajación también ayudan a manejar el estrés emocional. Diferentes terapias complementarias y alternativas como acupuntura, masajes, Tai chi, suplementos nutricionales, etc también han sido reportadas aunque su eficacia no está plenamente corroborada.

\section{Manejo farmacológico}

El tratamiento de la FM ha incluido durante buen tiempo el uso de antidepresivos puesto que ansiedad y depresión son frecuentes en estos pacientes, también porque diferentes antidepresivos han mostrado efecto analgésico en varias enfermedades que causan dolor crónico (19-21).

Entre los antidepresivos más usados está la amitriptilina (tricíclico), existen varios estudios ue sugieren su eficacia; sin embargo estos estudios son mayormente antiguos y sin una metodología adecuada por lo que actualmente se cree que si bien puede ser útil, su eficacia probablemente ayude a una baja proporción de pacientes (22).

Otros antidepresivos que también han mostrado cierta eficacia en el manejo de condiciones del dolor crónico, entre ellas FM son los inhibidores selectivos de la recaptación de serotonina, como Fluoxetina, Paroxetina, Sertralina (20,23-25), no obstante ello su eficacia no es convincente. Se supone que en los pacientes que no responden a estos medicamentos probablemente la norepinefrina juegue un rol importante en el control del dolor y por ello recientemente se han reportado estudios con los inhibidores duales de la recaptación de serotonina y norepinefrina que han mostrado eficacia en el manejo del dolor crónico en FM, entre ellos la Duloxetina y Minalcipran han sido recientemente aprobados por la Food and Drug Administration (FDA) de EEUU (26). Duloxetina, que también ha mostrado eficacia para el manejo de dolor crónico, dolor neuropático en polineuropatía diabética, lumbalgia refractaria a la terapia convencional (27), mostró en dos estudios importantes su eficacia en la reducción del dolor en FM $(28,29)$. Minalcipran es otro antidepresivo inhibidor dual de la recaptación de serotonina y norepinefrina que también se ha aprobado por la FDA de EEUU para el manejo del dolor en FM, al igual que duloxetina los efectos adversos más comunes son náuseas $\mathrm{y}$ constipación $(26,30)$.

Otros medicamentos usados para el manejo del dolor en FM son la Gabapentina y la Pregabalina. Aunque un estudio con Gabapentina a dosis de 1 $800 \mathrm{mg} /$ día mostró eficacia superior al placebo en la reducción del dolor (31), aún no está aprobada para el manejo de FM. A diferencia de ésta, la Pregabalina sí ha sido aprobada para el tratamiento de FM por la FDA de EEUU $(26,32)$; a dosis de 300-450 mg/día ha mostrado eficacia en la reducción del dolor, siendo 
sus principales efectos adversos son somnolencia y mareos (33).

Los anti-inflamatorios no esteroideos (AINE), analgésicos no opiáceos como paracetamol y opiáceos como tramadol, son usados para el manejo del dolor en estos pacientes. Asimismo, algunos ansiolíticos han sido usados también para mejorar el insomnio de algunos pacientes; sin embargo aunque ninguno de los fármacos mencionados han sido aprobados para el manejo de FM pueden ser de utilidad en algunos casos $(34,35)$. También diferentes relajantes musculares han sido usados en estos pacientes, entre ellos la ciclobenzaprina ha mostrado en algunos estudios reducción del dolor en estos pacientes (36).

Debe mencionarse que FM es una condición que cada día se reconoce más, probablemente por la gran carga de estrés laboral, familiar, social, a los que están sometidos los pacientes. Un diagnóstico apropiado y el uso racional de diferentes modalidades terapéuticas no farmacológicas como farmacológicas permiten un manejo eficaz del dolor y los demás síntomas asociados como ansiedad, depresión, insomnio, etc., mejorando así la calidad de vida de estos pacientes y permitiendo una rápida reinserción de ellos en el ambiente familiar, laboral y social.

\section{Declaración de conflictos de intereses:}

El autor declara no tener conflictos de intereses.

\section{Correspondencia:}

Alfredo Berrocal Kasay

Correo electrónico: alfredo.berrocal@upch.pe

\section{REFERENCIAS BIBLIOGRÁFICAS}

1. Wolfe F. Smythe H, Yunus M, et al. The ACR 1990 criteria for the classification of fibromyalgia. Report of the multicenter criteria committee. Arthritis Rheum. 1990; 33: 160-72.

2. Wolfe F, Claw D, Fitzcharles M, et al. The American College of Rheumatology preliminary diagnostic criteria for fibromyalgia and measurement of symptom severity. Arthritis Care Res. 2010; 62: 600-10.

3. Wolfe F, Ross K, Anderson J, et al. The prevalence and characteristics of fibromyalgia in the general population. Arthritis Rheum. 1995; 38: 19-28.

4. Aguilar J, Sánchez E, Salcedo C, et al. Estudio poblacional de frecuencia de enfermedades reumáticas en adultos mayores de 57 años. Boletín
Asoc Per Reumatol. 1994; I: 3-21.

5. Gursoy S, Erdal E, Herken H, et al. Association of T102C sm of the 5-HT2A receptor gene with psychiatric status in fibromyalgia syndrome. Rheumatol Int. 2001; 21: 58-61.

6. Gursoy S. Absence of association of the serotonin transporter gene polymorphism with the mentally healthy subset of fibromyalgia patients. Clin Rheumatol. 2002, 21. 194-7.

7. Dailey P, Bishop G, Russell I, et al. Psychological stress and the fibrositis/fibromyalgia syndrome. J Rheumatol. 1990; 17: 1380-5.

8. Imbierowicz K, Egle U. Childhood adversities in patients with fibromyalgia and somatoform pain disorder. Eur J Pain. 2003; 7: 113-9.

9. Bennett R. Emerging concepts in the neurobiology of chronic pain; evidence of abnormal sensory processing in fibromyalgia. Mayo Clin Proc. 1999; 74: 385-98.

10. Sumpton J, Moulin D. Fibromyalgia. Handb Clin Neurol. 2014; 119: 513-27.

11. Loggia M, Berna C, Kim J, et al. Disrupted brain circuitry for pain related reward/punishment in Fibromyalgia Arthr Rheum. 2014; 66: 203-12.

12. Rehm S, Koroschetz J, Gockel E, et al. A cross sectional survey of 3035 patients with fibromyalgia: subgroups of patients with typical comorbidities and sensory symptom profiles. Rheumatology (Oxford). 2010; 49: 1146-52.

13. Clauw D. Fibromyalgia: An overview. Am J Med. 2009; 122(S1): S3-S13.

14. Chung J, Kin S, Lee H, et al. The association between overactive bladder and Fibromyalgia syndrome: a community survey. Neurourol Urodyn. 2013; 32: 66-9.

15. Donmez S, Pamuk O, Umit E, Top M. Autoimmune rheumatic disease associated symptoms in fibromyalgia patients and their influence on anxiety, depression and somatization. A comparative study. Clin Exp Rheumatol. 2012; 30(S4): 65-9.

16. Hassett A, Gevirtz R. Non pharmacologic treatment for Fibromyalgia: patient education, cognitive behavioral therapy, relaxation techniques and medicine. Clin Rheum Dis. 2009; 35: 393-407.

17. Goossens M, Rutten van Molken M, Leidl R, et al. Cognitive educational treatment of fibromyalgia: a randomized clinical trial. I. Clininical effects. J Rheumatol. 1996; 23: 1237-45.

18. Williams D, Cary M, Groner K, et al. Improving physical functional status in patients with fibromyalgia: a brief cognitive behavioral intervention. J Rheumatol. 2002; 29: 1280-6.

19. Hudson J, Goldenberg D, Pope H, et al. Comorbidity of fibromyalgia with medical and psychotic disorders. Am J Med. 1992; 92: 363-7.

20. Goldenberg D, Maiskly M, Mossey C, et al. A randomized double blind cross over trial of fluoxetine 
and amitryptiline in the treatment of fibromyalgia. Arthr Rheum. 1996; 39: 1852-9.

21. Fishbain D. Evidence based data on pain relief with antidepressants. Ann Med. 2000; 32: 305-16.

22. Moore RA, Derry S, Aldington D, et al. Amitriptyline for neuropathic pain and fibromyalgia in adults. Cochrane Database Syst Rev 2012; 12:CD008242.

23. Wolfe F, Cathey M, Hawley D, et al. A double blind placebo controlled trial of fluoxetine in fibromyalgia. Scand J Rheumatol. 1994; 23: 255-9.

24. Patkar A, Masand P, Krulewicz S, et al. A randomized controlled trial of controlled release paroxetine in fibromyalgia. Am J Med. 2007; 120: 448-54.

25. Keefer F, Shelby R, Somers T, et al. Effects of coping skills training and Sertraline in patients with noncardiac chest pain: a randomized controlled study. Pain. 20112; 152: 730-41.

26. Nickerson B. Recent advances in the treatment of pain associated with Fibromyalgia. US Pharmacist. 2009; 34: 49-55.

27. Lunn M, Hughes R, Wiffen P. Duloxetin for treating painful neuropathy, chronic pain or fibromyalgiaCochrane Database Syst Rev 2014; 1: CD007115.doi : 10.1002 / 14651858.CD007115. pub3

28. Arnold L, Lu Y, Crofford 1, et al. A double blind multicenter trial comparing duloxetine with placeboin the treatment of fibromyalgia patients with or without major depressive disorders. Arthr Rheum. 2004; 50: 2974-84.

29. Arnold L, Rosen A, Pritchett Y, et al. A randomized double blind placebo controlled trial of duloxetine in the treatment of women with fibromyalgia with or without major depressive disorder. Pain. 2005; 119: 5-15.

30. Mase P, Clauw D, Gendreau M, et al. The efficacy and safety of minalcipran for the treatment of fibromyalgia: A randomized double blind placebo controlled trial. J Rheumatol. 2009; 36: 398-409.

31. Arnold D, Goldenberg D, Stanford S, et al: Gabapentin in the treatment of fibromyalgia. A randomized double blind placebo controlled multicenter trial. Arthritis Rheum2007; 56: 1336-44.

32. Crofford L, Rowbotham M, Mase P, et al. Pregabalin for the treatment of fibromyalgia syndrome. Results of a randomized double blind placebo controlled trial. Arthritis Rheum. 2005; 52: 1264-73.

33. Lyeseng-Williamson K, Siddiqui M. Pregabalin: A review of its use in Fibromyalgia. Drugs. 2008; 68: 2205-23.

34. Goldenberg D, Burckhardt C, Crofford L. Management of Fibromyalgia Syndrome. JAMA. 2004; 292: 2388-95.

35. Di Franco M, Iannuccelli C, Atzeni F, et al. Pharmacologic treatment of Fibromyalgia. Clin Exp Rheumatol. 2010; 28(S3): S 110-6.

36. Cimolai N. Cyclobezaprine: A new look at an old pharmacologic agent. Expert Rev Clin Pharmacol. 2009; 2: 255-63.

Recibido: 20/05/2014

Aceptado: 09/06/2014 